\title{
Symptomatic Extensive Thoracolumbar Epidural Hematoma Following Lumbar Disc Surgery Treated by Single Level Laminectomy
}

\author{
Youssef Ali $^{1,2}$, François-Xavier Roux ${ }^{1,2}$, Jean-François Meder ${ }^{2,3}$, Johan Pallud ${ }^{1,2}$ \\ ${ }^{1}$ Department of Neurosurgery, Sainte-Anne Hospital, Paris, France \\ ${ }^{2}$ University Paris Descartes, Paris, France \\ ${ }^{3}$ Department of Neuroradiology, Sainte-Anne Hospital, Paris, France
}

\begin{abstract}
Spinal epidural hematomas (SEHs) are rare complications following spine surgery, especially for single level lumbar discectomies. The appropriate surgical management for such cases remains to be investigated. We report a case of an extensive spinal epidural hematoma from T11-L5 following a L3-L4 discectomy. The patient underwent a single level L4. A complete evacuation of the SEH resulted in the patients full recovery. When presenting symptoms limited to the initial surgical site reveal an extensive postoperative $\mathrm{SEH}$, we propose: to tailor the surgical exposure individually based on preoperative findings of the SEH; and to begin the surgical exposure with a limited laminectomy focused on the symptomatic levels that may allow an efficient evacuation of the SEH instead of a systematic extensive laminectomy based on imaging.
\end{abstract}

Key Words: Epidural, Hematoma, Spine, Surgery, Management, Emergency, Postoperative

\section{Introduction}

Spinal epidural hematomas (SEHs) are rare but classic complications of lumbar disc surgery [1-4]. They are believed to originate from the venous plexus of the epidural space and are generally limited to the surgical site [5]. SEH must be suspected systematically in postoperative spine patients with acute symptoms of neurologic deterioration. An emergency surgical intervention is usually required. The most appropriate procedure, especially when the SEH extends beyond the initial surgical exposure, is not known. In such cases, a laminectomy extending as far as the SEH is generally performed [5]. We present a case of an extensive SEH from T11-L5 following a L3-L4 discectomy that presented with bilateral L4 radiculopathies at 8 hours postsurgery.

\section{Case Report}

A 46-year-old man, employed as a bricklayer, presented with a chief complaint of progressive left lower limb radiculopathy (visual analog score, VAS; 6/10) of two months duration. The patient's past medical history was unremarkable, especially with no use of aspirin or anticoagulant drugs and no known comorbidities (hypertension, vascular problems, diabetes, or coagulopathy). On physical preoperative examination, he showed a mild hypoesthesia in the left L4 dermatome. There was no bladder or erectile dysfunctions, and motor examination was normal. Radiographs of the lumbar spine did not reveal any significant abnormalities. Computed tomography of the lumbar spine demonstrated a left L3-L4 paramedian disc herniation (Fig. 1A and 1B). Lumbar resonance magnetic imaging was not performed.

The patient underwent a L3-L4 conventional microdis-

Received Jan 24, 2011; Revised May 8, 2011; Accepted May 12, 2011

Corresponding author: Johan Pallud, MD

Service de Neurochirurgie, Hopital Sainte-Anne,

1 rue Cabanis, 75674 Paris Cedex 14, France

Tel: +33-1-45 6584 87, Fax: +33-1-45 65 74 28, E-mail: johanpallud@hotmail.com

Copyright (C) 2012 by Korean Society of Spine Surgery which permits unrestricted non-commercial use, distribution, and reproduction in any medium, provided the original work is properly cited. Asian Spine Journal • pISSN 1976-1902 eISSN 1976-7846 
cectomy associated with a left L4 foraminotomy using a left intermyolaminar approach combined with a left interlaminar window in the genupectoral position under general anaesthesia. There was minimal blood loss, and no cerebrospinal fluid (CSF) leak was noted during surgery. No drain was inserted. At 2 hours postsurgery, the patient noted a complete resolution of the left L4 radiculopathy (VAS 1/10). At 8 hours postoperative, the patient complained of sudden-onset, severe pain radiating to the L4 dermatome (VAS 8/10) accompanied by numbness on both L4 dermatomes. Motor deficits were not observed. There was no bladder/bowel dysfunction. The postoperative magnetic resonance imaging
(MRI) demonstrated an epidural mass with an isointense signal on T1-weighted and a hypersignal on T2-weighted sequences; the mass extended from T11-L5, consistent with acute epidural haemorrhage and compression of the dural sac at the L3-L4 intervertebral disc level (Fig. 1C-1F). No signs of vascular malformations, including dural arteriovenous malformations, were shown on the MRI.

The patient underwent re-operation at 9 hours postsurgery. The patient was prepared for a laminectomy extending from T11 to L5 in a prone position under general anaesthesia. The surgical strategy began with a re-opening of the initial surgical site to allow a faster evacuation of the SEH.
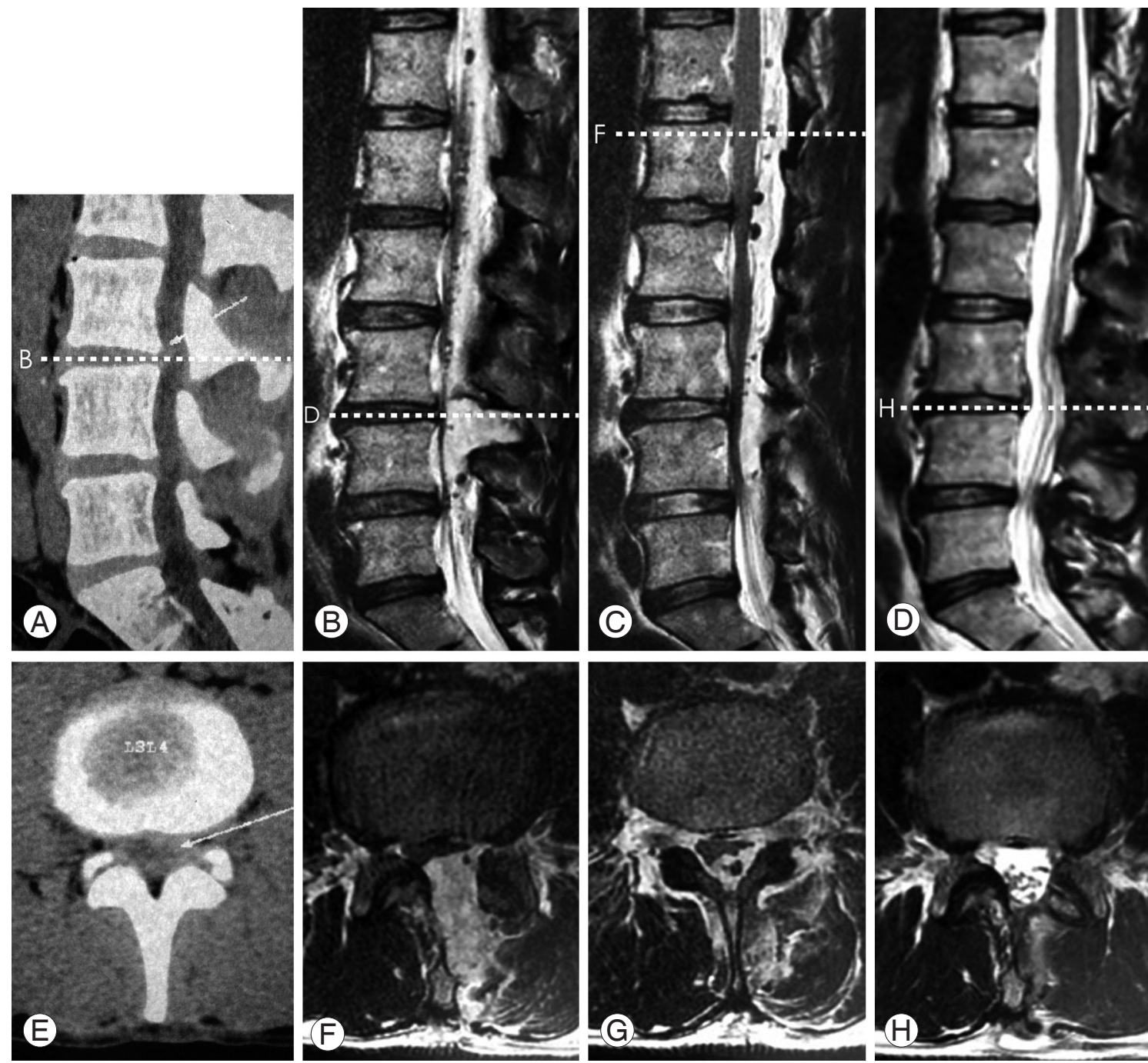

Fig. 1. (A, B) Preoperative lumbar CT-scan (A: sagittal image, B: axial image at the L3-L4 level) done 12 days prior to admission demonstrating a herniated disc at the level of the L3-L4 segment, with compression of the dural sac and the left L4 root. (C-F) Eight-hour postoperative magnetic resonance imaging (MRI) on T2weighted sequence (C, E: sagittal images, E, F: axial images) demonstrating an extensive T11 to L5 spinal epidural hematoma.. The dural sac compression is maximal at the surgical site (D) but is present at the upper levels (F). (G, H) Two-month postoperative MRI on T2-weighted sequence (G: sagittal image, H: axial image) demonstrating a complete evacuation of the spinal epidural hematoma with a dural sac. 
A limited L4 left hemi-laminectomy was additionally performed, allowing the spontaneous expulsion of a mature blood clot. The dural sac expanded completely within the vertebral canal, and no CSF leak was noted. In addition, an epidural drain ascending to the upper lumbar levels for $5 \mathrm{~cm}$ was blindly introduced.

At 2 hours postsurgery, the patient presented a complete resolution of the bilateral L4 radiculopathy and numbness. Motor deficits and no bladder/bowel dysfunction were noted. The drain, opened under gravity, drained $100 \mathrm{ml}$ of hematic fluid and was removed 24 hours postsurgery. The postoperative coagulopathy work-up showed no clotting disorder. No postoperative imaging was performed. The patient was discharged two days post-surgery. At two months, the neurologic examination was normal, MRI demonstrated no residual SEH, and the coagulopathy work-up was normal (Fig. $1 \mathrm{G}$ and $1 \mathrm{H}$ ).

\section{Discussion}

The reported incidence of postoperative SEH following spine surgery ranges from $0.1 \%$ to $0.2 \%[1-4]$. They are generally asymptomatic and confined to the surgical site, and symptomatic extensive thoraco-lumbar SEH are rarely reported [2]. Several risk factors have been identified, such as inadequate haemostasis before closure, multilevel procedures, age older than 60 years, a history of surgery at the same level, preoperative coagulopathy, liver and autoimmune diseases, heparinization after surgery, and hypotension during closure $[1,4,6-8]$. Our patient presented none of these risk factors.

In most cases, neurologic deficits are exceptional and, if present, are limited to the operated levels [9]. A MRI should be performed in postoperative spine patients with acute symptoms of neurologic deterioration [4,7]. The sagittal MRI usually demonstrates the extent of the SEH in the epidural space. The MRI features are highly suggestive of an acute haemorrhage with an isointense signal on T1weighted and a hypersignal on T2-weighted sequences, as in the present case [5]. Sometimes, a curvilinear band of hypersignal on a T1-weighted sequence that represents the epidural fat is observed between the SEH and the dural sac and may help in differentiating between disk protrusion and SEH [10].

Rapid surgical evacuation of the SEH is a determinant factor for a complete resolution of symptoms and a full neurologic recovery $[2,5,7]$. It has been shown that the time interval to evacuation of patients with a full recovery was shorter than in patients with an incomplete recovery [5]. In the present case, the SEH was evacuated at 9 hours postsurgery, i.e., 1 hour after its clinical revelation that allowed a full patient recovery, in accordance with previous reports. A neurologic improvement is classically observed after evacuation of postoperative SEH. However, overall functional outcomes are poorer as compared to patients without SEH [9]. Several factors may account for this: 1) the large surgical exposure during the second surgery may delay the patient's recovery, 2) the acute and transient nerve root compression may possibly prevent initial recovery of the nerve damage, and 3) the possible development of a postoperative fibrosis secondary to the SEH and to the surgical exposure, as demonstrated on postoperative imaging by an abnormal dural sac expansion. We suggest that a limited surgical exposure, whenever feasible, may reduce postoperative fibrosis, thus leading to a better outcome.

\section{REFERENCES}

1. Awad JN, Kebaish KM, Donigan J, Cohen DB, Kostuik JP. Analysis of the risk factors for the development of post-operative spinal epidural haematoma. J Bone Joint Surg Br 2005;87:1248-52.

2. Di Lauro L, Poli R, Bortoluzzi M, Marini G. Paresthesias after lumbar disc removal and their relationship to epidural hematoma. Report of two cases. J Neurosurg 1982;57:135-6.

3. Lawton MT, Porter RW, Heiserman JE, Jacobowitz R, Sonntag VK, Dickman CA. Surgical management of spinal epidural hematoma: relationship between surgical timing and neurological outcome. J Neurosurg 1995;83:1-7.

4. Uribe J, Moza K, Jimenez O, Green B, Levi AD. Delayed postoperative spinal epidural hematomas. Spine J 2003;3:125-9.

5. Yi S, Yoon do H, Kim KN, Kim SH, Shin HC. Postoperative spinal epidural hematoma: risk factor and clinical outcome. Yonsei Med J 2006;47:326-32.

6. Kou J, Fischgrund J, Biddinger A, Herkowitz H. Risk factors for spinal epidural hematoma after spinal surgery. Spine (Phila Pa 1976) 2002;27:1670-3.

7. Lagares A, González P, Rivas JJ, Lobato RD, Ramos A. Epidural haematoma after lumbar disc surgery causing radiculopathy. Acta Neurochir (Wien) 1999;141:123940. 
8. Spanier DE, Stambough JL. Delayed postoperative epidural hematoma formation after heparinization in lumbar spinal surgery. J Spinal Disord 2000;13:46-9.

9. Ikuta K, Tono O, Tanaka T, et al. Evaluation of postoperative spinal epidural hematoma after microendoscopic posterior decompression for lumbar spinal stenosis: a clinical and magnetic resonance imaging study. J Neurosurg Spine 2006;5:404-9.

10. Dorsay TA, Helms CA. MR imaging of epidural hematoma in the lumbar spine. Skeletal Radiol 2002;31:67785. 